\title{
CAZUMA 1, NUEVA LOCALIDAD DE MICROMAMÍFEROS (MAMMALIA, RODENTIA) DEL ARAGONIENSE SUPERIOR EN LA CUENCA DE QUESA - BICORP (PROV. VALENCIA)
}

\author{
Francisco J. RUIZ-SÁNCHEZ1, Carlos DE SAN- \\ TISTEBAN $N^{1}$ y José I. LACOMBA ${ }^{2}$ \\ ${ }^{1}$ Departament de Geologia. Universitat de València. C/ Dr. Moliner, s/n. 46100 \\ Burjassot. València. \\ ${ }^{2}$ Conselleria de Territori i Habitatge. Generalitat Valencia. C/ Gregorio Gea, 26. \\ E-mail: francisco.ruiz@uv.es \\ E-mail: carlos.santisteban@uv.es \\ E-mail: ignacio.lacomba@cma.m400.gva.es
}

Ruiz-Sánchez, F. J., Santisteban, C. de \& Lacomba, J. I. 2005. Cazuma 1, nueva localidad de micromamíferos (Mammalia, Rodentia) del Aragoniense superior en la cuenca de Quesa - Bicorp (prov. Valencia). [Cazuma 1, new micromammal locality (Mammalia, Rodentia) of late Aragonian of the Quesa - Bicorp basin (Valencia, Spain).] Revista Española de Paleontología, N.E. X, 101-109. ISSN 0213-6937.

\begin{abstract}
The association of Megacricetodon crusafonti (Freudenthal, 1963), Megacricetodon sp. and Heteroxerus aff. grivensis (Forsyth Major, 1893) of the Cazuma 1, fossil site in the area of Bicorp (Quesa-Bicorp basin, southern part of the Valencia Province), is here described. The assigned age for this site is late Aragonian. Up to present, five Aragonian fossil sites have been found in this basin: Cazuma 1, Quesa 0, Quesa 2, Quesa 3 and Quesa 6.
\end{abstract}

Keywords: Rodentia, Aragonian, Quesa - Bicorp basin, Valencia.

\section{RESUMEN}

En el presente trabajo se describe la asociación de Megacricetodon crusafonti (Freudenthal, 1963), Megacricetodon sp. y Heteroxerus aff. grivensis (Forsyth Major, 1893) de Cazuma 1, localidad situada en el sector de Bicorp, en la cuenca terciaria de Quesa - Bicorp (Prov. Valencia). La edad atribuida a esta localidad es Aragoniense Superior. Hasta el momento, con ésta, ya son cinco las localidades conocidas de mamíferos en el Aragoniense de la cuenca de Quesa - Bicorp (Cazuma 1, Quesa 0, Quesa 2, Quesa 3 y Quesa 6).

Palabras claves: Rodentia, Aragoniense, cuenca de Quesa - Bicorp, Valencia.

\section{INTRODUCCIÓN}

La cuenca de Quesa - Bicorp pertenece al conjunto de las cuencas terciarias levantinas, emplazadas mayormente en el Sistema Ibérico y en el área de transición entre éste y las Béticas. Estas cuencas son muy diversas en tamaño, espesor y naturaleza de su registro sedimentario, aunque presentan similitudes en su evolución tectosedimentaria (Santisteban et al., 1987). La mayor parte de ellas fueron fosas tectónicas con orientaciones NNO - SSE, NE - SO e incluso E - O. Globalmente la extensión de sus afloramientos varía entre 2.500 y $14 \mathrm{~km}^{2}$, y la potencia de su registro oscila entre menos de $200 \mathrm{~m}$ y los $800 \mathrm{~m}$. Éste está forma- do por dos tramos, separados por una discordancia angular de extensión regional (Santisteban et al., 1987). El tramo inferior abarca un intervalo de edad Cretácico superior - Oligoceno, mientras que el tramo superior es Mioceno inferior - Plioceno. Las cuencas emplazadas en el ámbito del Sistema Ibérico presentan afloramientos con materiales de los dos tramos, estando los depósitos del tramo inferior afectados por las estructuras de deformación alpinas. Los datos de los que se dispone hasta hoy (Gutiérrez et $a l ., 1984)$, indican que las cuencas emplazadas en la zona de transición Ibérica - Béticas (Ayora - Cofrentes, Quesa - Bicorp y Navarrés) presentan un registro formado exclusivamente por depósitos del tramo superior. De ellas, la de 
Quesa - Bicorp tiene un espesor de sedimentos cercano a los $750 \mathrm{~m}$, en facies deposicionales aluviales y lacustres, distribuídos en dos sectores separados por afloramientos de materiales del Triásico superior. Según Roca et al. (1996), la removilización diapírica de los depósitos de las facies Keuper, en Bicorp, es sinsedimentaria y consecuencia de la última fase de plegamiento Bético.

En los sectores de Quesa y Bicorp han sido prospectadas varias áreas ante la posibilidad que contuvieran restos de micromamíferos fósiles. Ello ha permitido localizar cerca de una decena de puntos de muestreo favorables. En el área de Bicorp se han muestreado las series del Río Cazuma y Barranco de Moreno. En el presente trabajo se describe el conjunto faunístico del yacimiento Cazuma 1, localizado en la serie del Río Cazuma.

\section{SITUACION GEOLOGICA}

Originalmente la cuenca de Quesa - Bicorp tuvo una extensión de $50 \mathrm{~km}^{2}$ y una configuración alargada en la dirección NE - SO (Fig. 1). Fue una cuenca subsidente, emplazada en una fosa tectónica en la que se formó un registro de $750 \mathrm{~m}$ de espesor de materiales continentales (aluviales y lacustres) en un periodo de tiempo comprendido entre el Aragoniense medio y el Turoliense (Santisteban et al., 1994).

El yacimiento Cazuma 1 se encuentra localizado en el sector Norte de la cuenca Quesa - Bicorp (Fig. 1). Los depósitos terciarios de Quesa - Bicorp afloran fragmentariamente en tres sectores distintos: 1) valle de río Grande (Quesa), 2) valle del río Ludey (Quesa), y 3) valles de los ríos Cazuma y Bicorp (Bicorp), distinguiéndose tres unidades que son, brechas de margen de cuenca, depósitos aluviales, y un complejo lacustre (Santisteban et al., 1994).

En los depósitos aluviales es donde se encuentra situada la localidad Cazuma 1 (Fig. 2). Los depósitos del valle del río Cazuma están formados por $90 \mathrm{~m}$ de arcillas, areniscas y conglomerados rojos, que incluyen de 8 a 10 intercalaciones de margas y calizas laminadas palustres, de un espesor no superior a $7 \mathrm{~m}$ cada una. Hacia el norte (sectores de Ludey y Cazuma - Bicorp) estos materiales pasan a los depósitos basales del complejo lacustre, mediante un sistema de cuñas interdigitadas.

El nivel muestreado corresponde a una capa de margas oscuras ricas en materia orgánica y con abundantes restos de gasterópodos dulceacuícolas, intercalada entre calizas laminadas de origen orgánico (Fig. 3). Su localización en coordenadas U.T.M. es: 30XJ88603235.

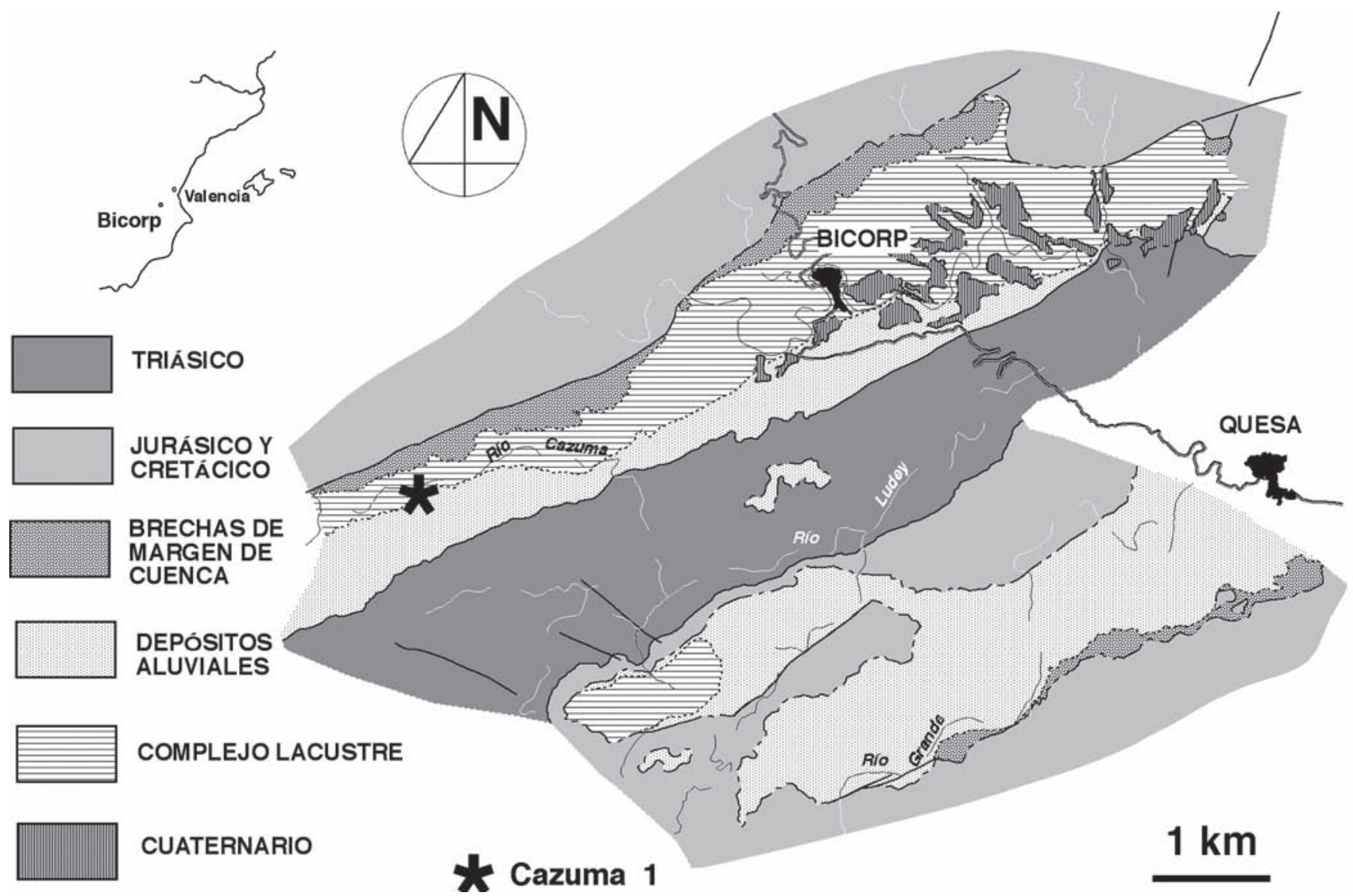

Figura 1. Mapa geológico de la cuenca de Quesa - Bicorp. Geological map of the Quesa - Bicorp basin. 


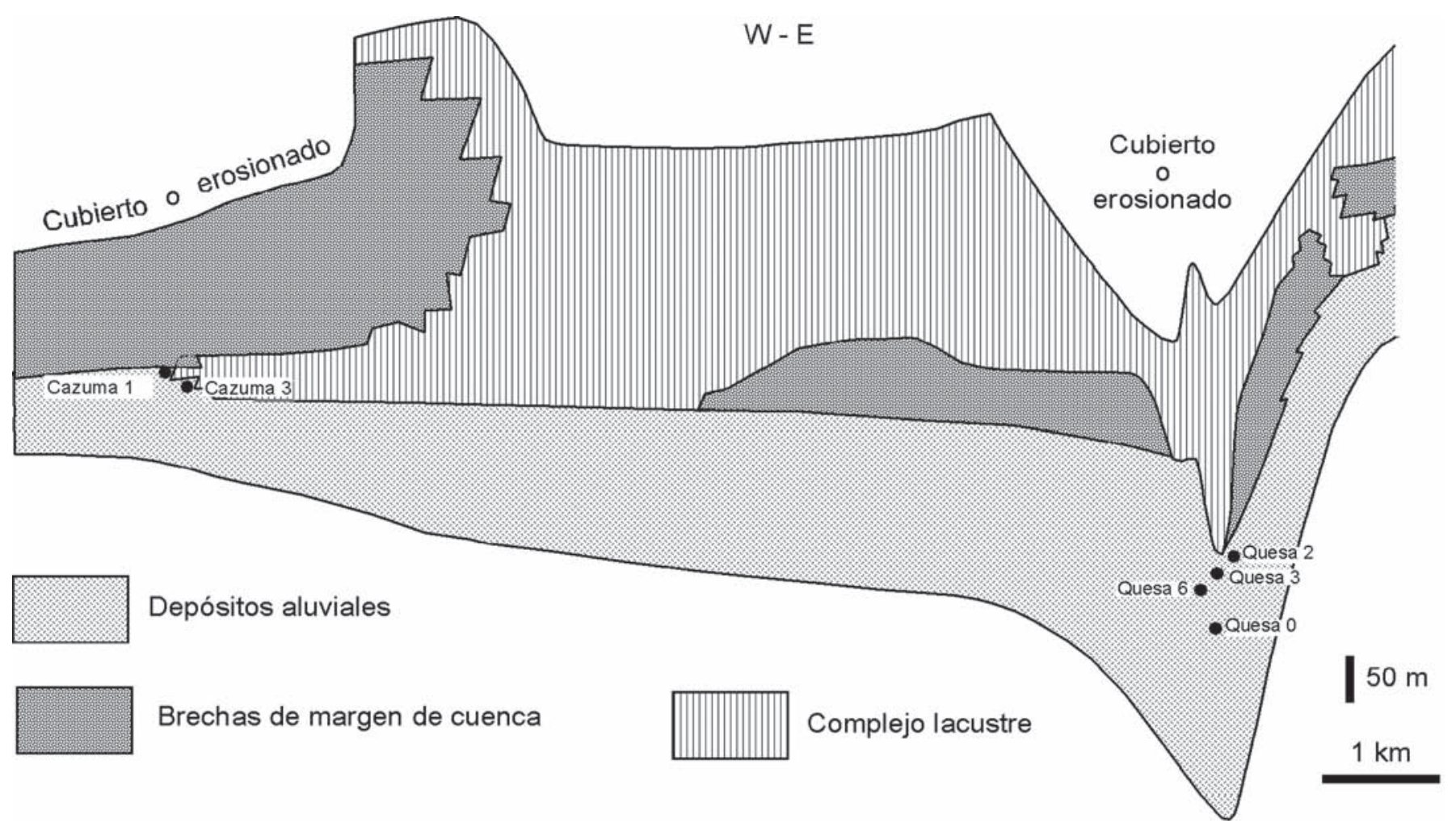

Figura 2. Esquema estratigráfico NE - SO de la cuenca de Quesa - Bicorp. Stratigraphic NE - SW cross - section of the Quesa - Bicorp basin.

\begin{tabular}{|c|c|c|c|c|c|c|c|c|}
\hline & \multicolumn{4}{|c|}{ LONGITUD } & \multicolumn{4}{|c|}{ ANCHURA } \\
\hline & $\mathbf{N}$ & mín & med & máx & $\mathbf{N}$ & mín & Med & Máx \\
\hline $\mathbf{M}_{1}$ & 7 & 15,5 & 16,2 & 17,3 & 7 & 9,4 & 10,1 & 10,7 \\
\hline $\mathbf{M}_{2}$ & 14 & 12,2 & 13,1 & 13,8 & 17 & 8,9 & 10,1 & 11,1 \\
\hline $\mathbf{M}_{3}$ & 6 & 9,7 & 10,3 & 10,7 & 6 & 8,1 & 8,6 & 9 \\
\hline $\mathbf{M}^{1}$ & 6 & 16,6 & 17,4 & 18,1 & 6 & 11,2 & 11,7 & 12,7 \\
\hline $\mathbf{M}^{2}$ & 7 & 11,2 & 12,5 & 13,7 & 6 & 10,1 & 10,6 & 11,2 \\
\hline $\mathbf{M}^{3}$ & 5 & 7,4 & 8,5 & 8,9 & 5 & 7,4 & 8,2 & 9 \\
\hline
\end{tabular}

Tabla 1. Tabla de medidas de Megacricetodon crusafonti (Freudenthal, 1963) de la localidad Cazuma 1. $\mathrm{N}$ : Número de dientes. mín: valor mínimo. med: media. máx: valor máximo. Las medidas se dan en décimas de milímetro.

Measurements of Megacricetodon crusafonti (Freudenthal, 1963) from Cazuma 1 locality. N: teeth number. mín: minimal value. med: mean. máx: maxim value. Measurements are given in 0,1 mm units.

\section{MATERIAL Y MÉTODOS}

De la localidad Cazuma 1 se ha extraído 2.500 kg de sedimento que, una vez procesado y triado, ha proporcionado una colección de cincuenta restos fósiles de roedores de las familias Cricetidae y Sciuridae.

Las descripciones y medidas de las piezas se han realizado utilizando una lupa binocular Wild dotada de regleta ocular de medida. La nomenclatura usada para la descripción del material del género Megacricetodon Fahlbusch, 1964 ha sido tomada de Daams \& Freudenthal (1988), y para los de la familia Sciuridae la de Cuenca (1988).

El material que compone la colección de la localidad Cazuma 1 se encuentra depositado bajo las siglas CAZ1- en el Museo del Departament de Geologia de la Universitat de València.

\section{PALEONTOLOGÍA SISTEMÁTICA}

ORDEN RODENTIA Bodwich, 1821

Familia Cricetidae Rochebrune, 1883

Género Megacricetodon Fahlbusch, 1964

Especie tipo: Cricetodon minor Lartet, 1851 


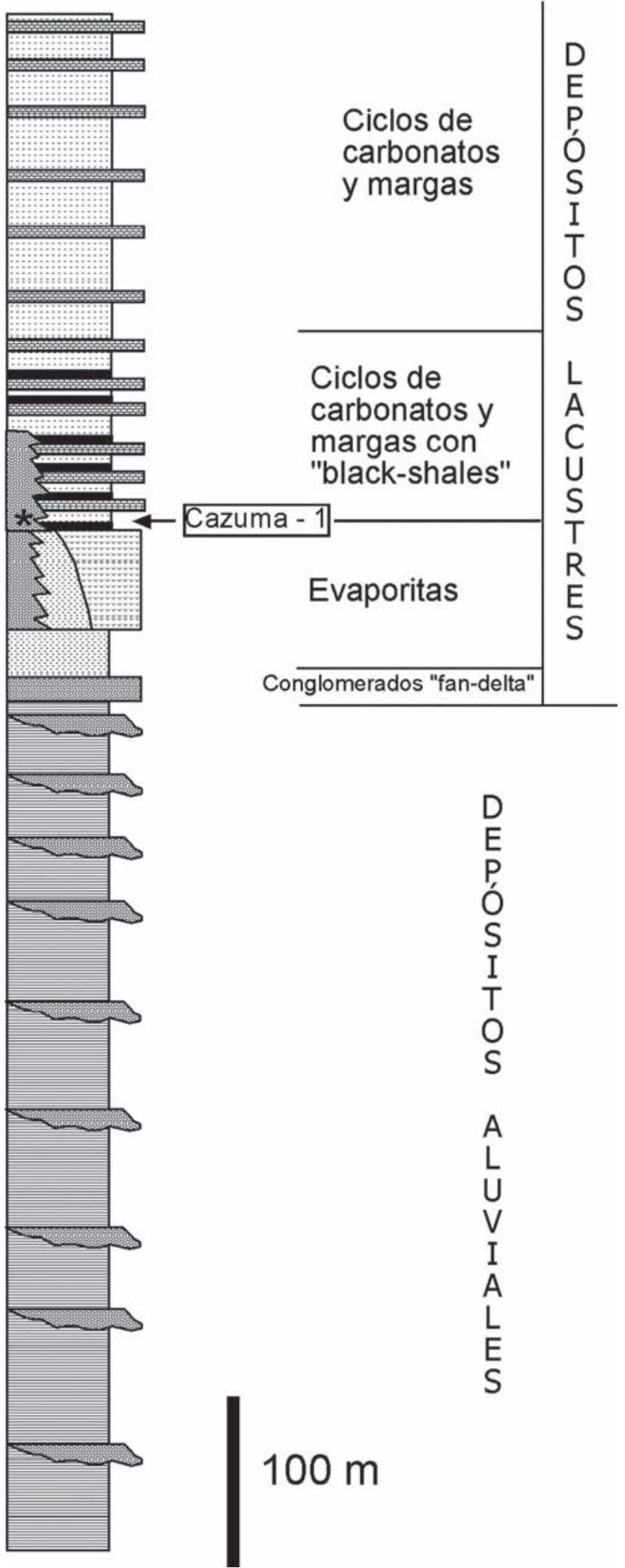

Figura 3. Columna estratigráfica de la sección del Río Cazuma. La posición de la localidad Cazuma 1 se indica mediante asterisco.

Stratigraphic column of Cazuma river section. Position of Cazuma 1 locality is indicated by an asterisk.

\section{Megacricetodon crusafonti (Freudenthal, 1963)} Fig. 5a-5j

Material y medidas: Ver tabla 1.

\section{Descripción:}

M1: Dientes de contorno subtriangular. Anterocónido grueso, puntiforme (1) o dividido (3). Mesolófido ausente.

M2: Dientes de contorno subcuadrangular - subrectangular. Anterolófido lingual ausente (9) o muy pequeño (4). Mesolófido ausente (9), muy corto (7) o corto (2).

M3: Dientes de contorno subtrapezoidal. Anterolófido lingual muy corto (4) o de mediana longitud (1). Mesolófido ausente. Hipolofúlido corto o muy corto. Sénido poco profundo.

M1: Dientes de contorno subrectangular. Plataforma anterior grande (5) o ausente (1). Espolones labial y lingual del anterolófulo ausentes. Ectolofo ausente (2), muy corto (2) o de mediana longitud (2). La unión ectolofo - mesolofo se observa en un sólo caso. Mesolofo muy corto (2) o corto (5). Posteroseno muy pequeño.

$\mathbf{M}^{2}$ : Dientes de contorno subrectangular o subcuadrangular. Protolófulo anterior (2), posterior (4) o doble (1). Mesolofo corto (6) o medio (1). Ectolofo medio o largo, no unido al mesolofo. Seno transversal (3) o anterior (3). Metalófulo anterior (2), ausente (1) o posterior (4).

$\mathbf{M}^{3}$ : Anterolofo lingual ausente (2) o muy corto (2). Protolófulo anterior. Anteroseno pequeño y estrecho. Neo - entolofo alto y continuo. Seno poco profundo. Axiolofo ausente. Centrolofo largo y continuo, excepto en un caso que está interrumpido. Mesolofo ausente (2) o corto (2).

\section{Discusión:}

El género Megacricetodon, en los yacimientos de micromamíferos de edad Aragoniense de la Península Ibérica, suele constituirse como el componente principal de las faunas de roedores. Las extensas colecciones de éste y otros géneros de roedores de casi un centenar de localidades de la cuenca de Calatayud - Daroca han permitido la confección de varias biozonaciones del Aragoniense (Daams \& Freudenthal, 1981, 1988; Daams et al., 1999).

En la localidad Cazuma 1 hemos podido caracterizar morfológica y biométricamente (Tabla 1 y Fig. 4) el material fósil. De su estudio se desprende que, por un lado la biometría del conjunto de elementos dentarios, y por otro la morfología del anterocónido y mesolófido del $\mathrm{M}_{1}$, anterolófido lingual del $\mathrm{M}_{2} \mathrm{y}$ protolófulo del $\mathrm{M}^{2}$ son similares a los de M. crusafonti (Freudenthal, 1963), de la biozona G2 del Aragoniense superior de Calatayud - Daroca (Daams \& Freudenthal, 1988; Daams et al., 1999).

Anterocónido M1: La estructura del anterocónido de los $\mathrm{M}_{1}$ se ha considerado como uno de los caracteres 
M1 inf

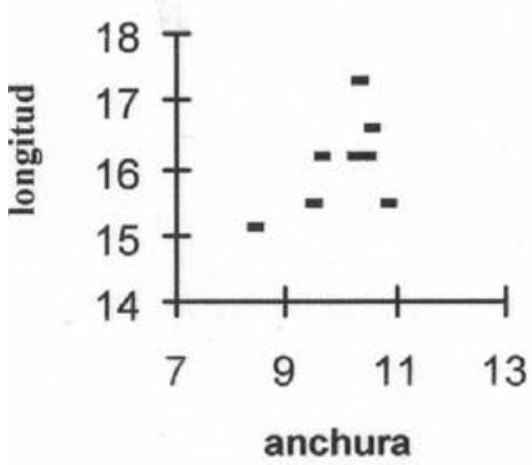

M1 sup

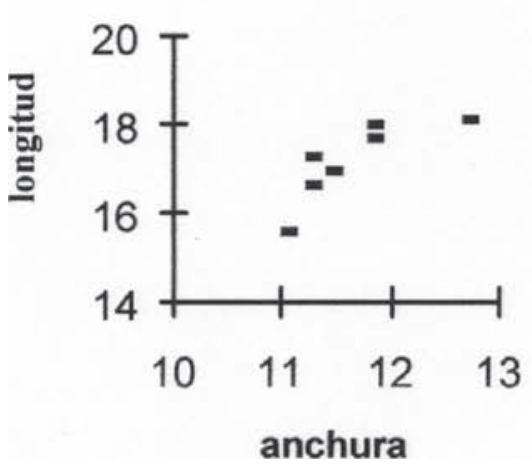

M2 inf

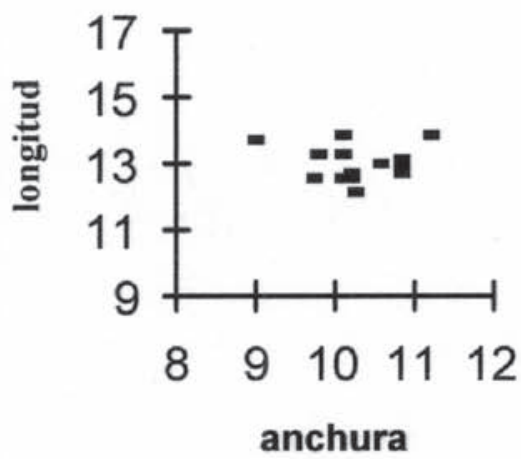

M2 sup

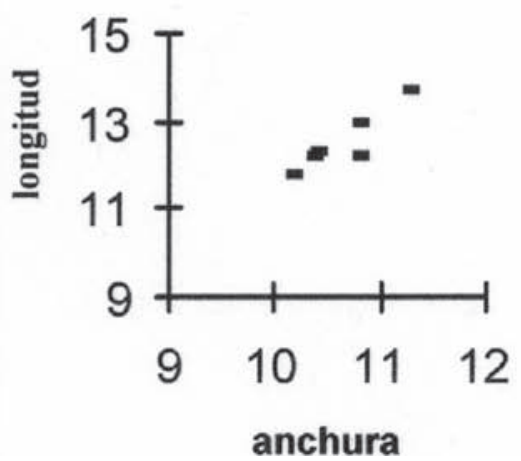

M3 inf

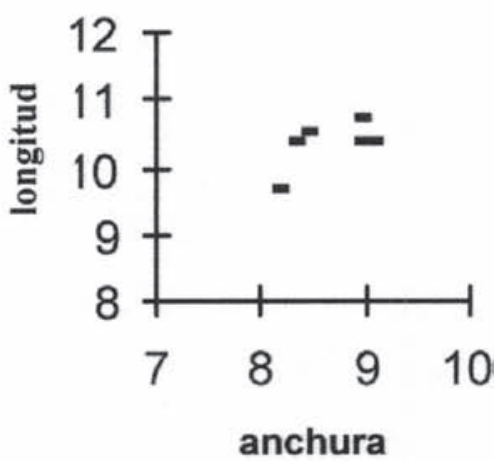

M3 sup

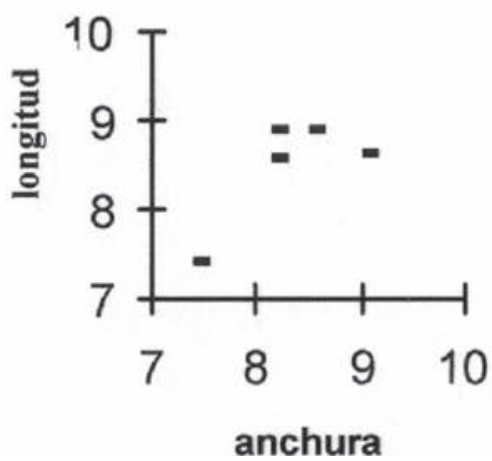

Figura 4. Diagramas Longitud/Anchura de los $\mathrm{M}_{1}, \mathrm{M}_{2}, \mathrm{M}_{3}, \mathrm{M}^{1}, \mathrm{M}^{2}$ y $\mathrm{M}^{3}$ de Megacricetodon crusafonti (Freudenthal, 1963) de Cazuma 1.

Length/Width scatter diagrams of the molars $\left(M_{1}, M_{2}, M_{3}, M^{l}, M^{2}\right.$ and $\left.M^{3}\right)$ of Megacricetodon crusafonti (Freudenthal, 1963) from Cazuma 1.

morfológicos de mayor relevancia para la determinación taxonómica de las diferentes especies del género Megacricetodon. En las localidades entre el Aragoniense inferior y el Vallesiense inferior se observa un progresivo aumento en la división de esta cúspide (Daams \& Freudenthal, 1988). Mientras las especies del género en el Aragoniense inferior se caracterizan por anterocónidos de tipo puntiforme, en M. ibericus (Schaub, 1944) del Vallesiense inferior, la práctica totalidad de ejemplares presentan un anterocónido profundamente dividido.

El anterocónido en la población de Cazuma 1 está dividido. Sólo uno de los ejemplares presenta un anterocónido puntiforme. Esta configuración del carácter es típica de los representantes del género a partir del Aragoniense medio. En M. collongensis (Mein, 1958) de la localidad Quesa 2 (Ruiz - Sánchez et al., 1995) son mayoritarias las morfologías con anterocónido puntiforme o ligeramente dividido.
Mesolófido M1: Daams \& Freudenthal (1988) estudian la variabilidad morfológica del mesolófido del $\mathrm{M}_{1}$ de $M e$ gacricetodon en las localidades del Aragoniense inferior - Vallesiense inferior de la cuenca de Calatayud - Daroca. De la variabilidad fenotípica observada, estos autores caracterizan cuatro tipos básicos de morfologías; el "morfotipo" 1, como aquel que presenta un mesolófido largo. El tipo 2, para los ejemplares que tienen un mesolófido medio, el tipo 3, para los que tienen un mesolófido corto, y el tipo 4, para los especimenes donde no existe mesolófido.

En Cazuma 1 la única morfología encontrada es la de tipo 4, esto es, aquella con mesolófido ausente. La ausencia de esta cresta es común en poblaciones del género de localidades del Aragoniense medio - superior de la cuenca de Calatayud - Daroca.

Anterolófido lingual $\mathbf{M}_{2}$ : El anterolófido lingual del $\mathrm{M}_{2}$ de la localidad Cazuma 1 no existe en nueve casos, y 


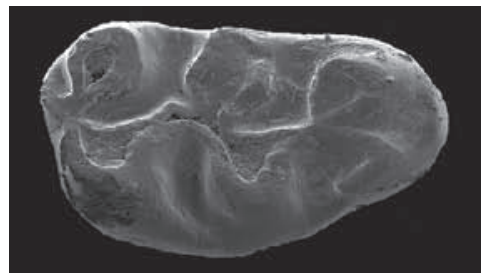

a

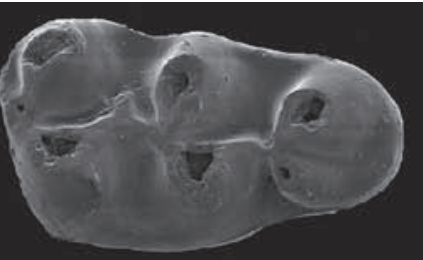

b

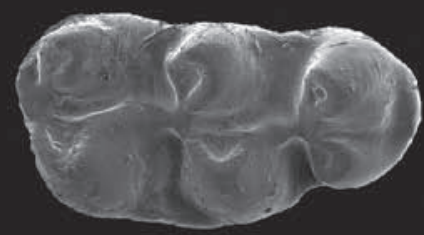

c

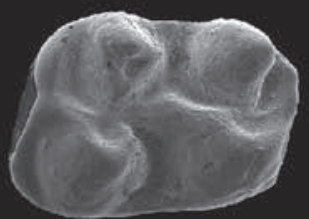

d

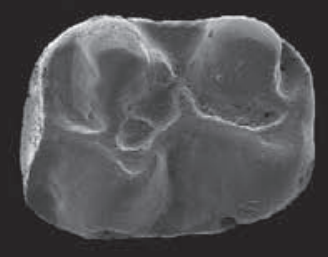

e

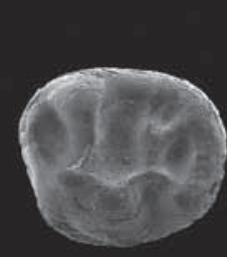

f

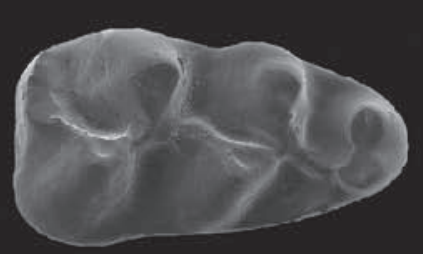

g

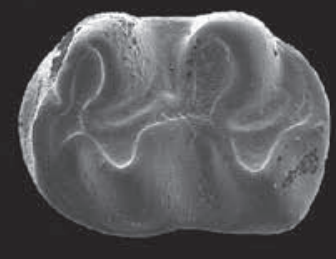

h

$1 \mathrm{~mm}$
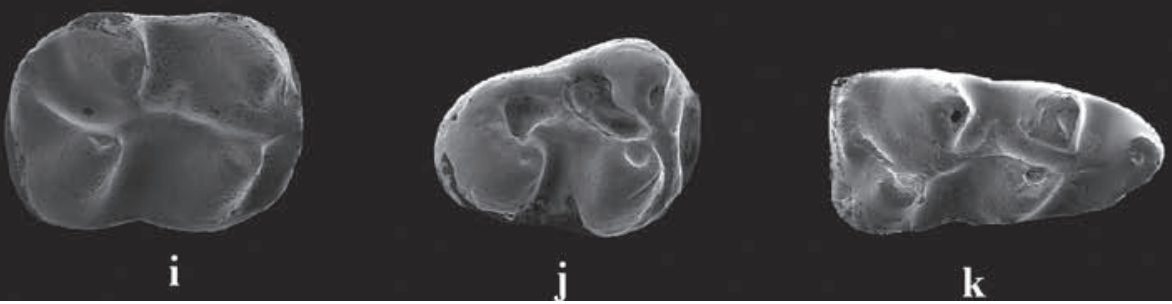

$\mathbf{k}$

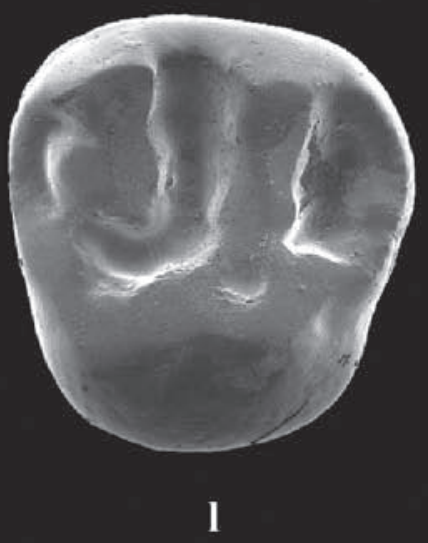

Figura 5. Megacricetodon crusafonti (Freudenthal, 1963). a - j. a, $\mathrm{M}^{1}$ dex., CAZ1-1; b, $\mathrm{M}^{1}$ dex., CAZ1-3; c, M1 dex., CAZ1-5;

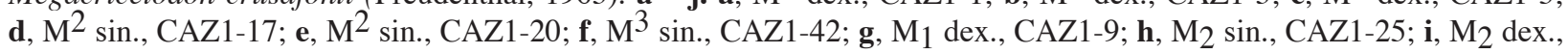
CAZ1-32; j, M3 dex., CAZ1-47. k. Megacricetodon sp., M1 dex., CAZ1-11. 1. Heteroxerus aff. grivensis (Forsyth Major, 1893), $\mathrm{P}^{4}$ sin., CAZ1-56.

Megacricetodon crusafonti (Freudenthal, 1963). $\boldsymbol{a}-\boldsymbol{j} . \boldsymbol{a}, M^{1}$ dex., CAZ1-1; b, $M^{1}$ dex., CAZ1-3; $\boldsymbol{c}, M^{1}$ dex., CAZ1-5; d, $M^{2} \sin ., C A Z 1-17 ; \boldsymbol{e}, M^{2} \sin ., C A Z 1-20 ; \boldsymbol{f}, M^{3} \sin ., C A Z 1-42 ; \boldsymbol{g}, M_{1}$ dex., CAZ1-9; $\boldsymbol{h}, M_{2}$ sin., CAZ1-25; $\boldsymbol{i}, M_{2}$ dex., CAZ1-32; j, $M_{3}$ dex., CAZ1-47. k. Megacricetodon sp., Ml dex., CAZ1-11. l. Heteroxerus aff. grivensis (Forsyth Major, 1893), $P^{4} \sin .$, CAZ1-56.

es muy corto en otros cuatro. Daams \& Freudenthal (1988) apuntan una progresiva disminución de la longitud de esta cresta en las diferentes poblaciones del género a lo largo del Aragoniense. El tipo y porcentaje de representación de las morfologías del anterolófido lingual del $\mathrm{M}_{2}$ en la localidad valenciana son, al igual que ocurre con el anterocónido y el mesolófido del $\mathrm{M}_{1}$, comunes en poblaciones del género Megacricetodon del Aragoniense medio - superior de Calatayud - Daroca.

Protolófulo del $\mathbf{M}^{2}$ : Quizás sea este carácter uno de los más relevantes para la determinación taxonómica de los representantes del género Megacricetodon del Aragonien- se medio, superior y del Vallesiense inferior (Freudenthal, 1963,1968; Ruiz - Sánchez, 1999).

La evolución de la morfología del protolófulo del $\mathrm{M}^{2}$ de Megacricetodon a lo largo del Aragoniense y Vallesiense inferior presenta una complicación extraordinaria. La morfología de este carácter parece tender hacia un aumento de la complejidad, traducida en un porcentaje mayor de protolófulos dobles, con o sin la rama posterior parcialmente interrumpida. También es de destacar la aparición de ejemplares con una conexión simple y directa entre metacono y entolofo.

Daams \& Freudenthal (1988) estudian el desarrollo del protolófulo del $\mathrm{M}^{2}$ del género Megacricetodon en 
los yacimientos del Aragoniense y Vallesiense inferior de Calatayud - Daroca, estableciendo cinco morfologías características. Estas son: MF1, con protolófulo simple y anterior, unido al anterolófulo, MF2, donde el protolófulo es simple y transversal, unido al protocono, MF3, en la que el protolófulo es simple y posterior, unido al entolofo, MF4, con un protolófulo simple y entolofo unido al paracono, y a su vez desconectado del protocono, y MF5, que posee un protolófulo doble.

Según estos mismos autores, en las localidades de las biozona C y D del Aragoniense de Calatayud - Daroca son mayoritarias, por este orden, las morfologías de tipo MF1, MF5 y MF2, con pequeños porcentajes del tipo MF4, mientras que en localidades de la biozona E (Las Planas 4A, 4B y 4C) empiezan a aparecer, además, ejemplares con morfologías de tipo MF3. En la zona F dominan las morfologías de tipo MF3, MF4 y MF5, reduciéndose mucho el porcentaje de aparición de la morfología MF1. Por último, en la zona $\mathrm{G}$ destaca, en relación a lo que ocurre en la zona F, la importante disminución de ejemplares con protolófulo doble (MF5).

La morfología de este carácter parece estar íntimamente ligada al desarrollo que adopte el seno. El mayor o menor redondeamiento de la parte posterior del $\mathrm{M}^{2}$ determina un seno posterior o transversal, respectivamente, constriñiendo, a su vez, el desarrollo del entolofo y las conexiones que puede establecer el protolófulo. Freudenthal (1963; 1968) establece una hipótesis sobre la evolución de este carácter. Según esta hipótesis, la morfología más primitiva sería aquella en que el protolófulo es simple y se encuentra conectado directamente al protocono, y la más moderna la de un protolófulo simple y dirigido hacia la parte posterior, en la que puede quedar unido al entolofo.

Una secuencia, estratigráficamente superpuesta, de localidades del Aragoniense inferior y medio en la cuenca del Río Magro nos ha permitido estudiar la variación morfológica de este carácter (Ruiz - Sánchez, 1999). El resultado de este análisis nos induce a considerar una evolución del carácter en el sentido: MF2 MF1 MF5 MF4 MF3, al igual que parece ocurrir en las poblaciones de Megacricetodon del Aragoniense y Vallesiense inferior de Calatayud - Daroca (Daams \& Freudenthal, 1988).

En la localidad Cazuma 1, el protolófulo es simple, anterior y unido al anterolófulo (MF1) en dos casos, simple, posterior, y unido al entolofo (MF3) en cuatro, y doble (MF5) en uno. Según nuestra interpretación, más del 50\% de casos corresponderían al tipo más moderno (MF3).

\section{Comparaciones con otras localidades:}

Las medidas de Megacricetodon crusafonti de Cazuma 1 se encuentran en el rango de variabilidad de la mayoría de localidades de la Península Ibérica donde aparece este taxón (Daams \& Freudenthal, 1988; Sesé et al., 1985). En general, la talla es algo superior en los casos de Póvoa de Santarém y Pero Filho en Portugal (Antunes \& Mein,
1977), e inferior en el material de la localidad de Villanueva de Huerva (Pérez et al., 1985), en la cuenca del Ebro. Los valores máximos de Villanueva de Huerva se encuentran por debajo de los mínimos de Cazuma 1.

La morfología de Megacricetodon crusafonti de Cazuma 1 es muy similar a la de esta especie en la localidad tipo de Manchones, así como a la de los yacimientos de la cuenca de Madrid, Paracuellos 3, Paracuellos 5 y Henares 1 (Sesé et al., 1985). Esta similitud está basada, fundamentalmente, en la existencia en las localidades madrileñas de M1 con anterocónidos divididos ligera o más profundamente, y de mesolófidos muy cortos o inexistentes.

En Fuen Vich-1, localidad de la cuenca del Cabriel, se ha determinado Megacricetodon crusafonti (ver Ruiz - Sánchez et al., 1994). Las dimensiones del M1 de Fuen Vich-1 son algo inferiores a las del $\mathrm{M}_{1}$ de Megacricetodon crusafonti de Cazuma 1. Morfológicamente difieren en cuanto al grado de división del anterocónido del $\mathrm{M}_{1}$. Así, en Fuen Vich-1 aparece un anterocónido profundamente dividido. El desarrollo del mesolófido es exactamente igual en ambas localidades, esto es, en ningún ejemplar el mesolófido se encuentra presente.

\section{Megacricetodon sp.}

Fig. 5k

Material y medidas: $\mathrm{Un}_{1} \mathrm{M}_{1}$ con una longitud de $1,51 \mathrm{~mm}$ y una anchura de $0,83 \mathrm{~mm}$.

Descripción: M1 diente de contorno subtriangular. Cúspides muy altas y ligeramente volcadas hacia la parte anterior. Anterocónido puntiforme. Mesolófido de medio a largo. Hipolofúlido anterior. Posterosénido grande.

\section{Discusión:}

En localidades del Aragoniense medio de la cuenca de Calatayud - Daroca aparecen juntas dos especies del género Megacricetodon (Daams \& Freudenthal, 1988), distintas en talla y morfología. En el Aragoniense medio - superior de la Península Ibérica se ha descrito especies del género Megacricetodon de talla reducida. Estas especies son: Megacricetodon rafaeli Daams \& Freudenthal, 1988, Megacricetodon minor (Lartet, 1851), Megacricetodon debruijni Freudenthal, 1968, Megacricetodon similis Fahlbusch, 1964, Megacricetodon lopezae García Moreno in Álvarez - Sierra \& García - Moreno, 1986, y Megacricetodon freudenthali García Moreno, 1986. Estos taxones se caracterizan, además de por su reducida talla respecto de los restantes taxones del género, por tener una morfología comparable a las formas de mayor tamaño del género Megacricetodon del Aragoniense inferior.

El ejemplar CAZ1-11 presenta el anterocónido puntiforme y el mesolófido largo. Las medidas de CAZ1-11 son demasiado grandes para poder ser atribuidas a Megacricetodon minor o Megacricetodon debruijni de Pedregue- 
ras IIC, Nombrevilla y Pedregueras IA, en el Vallesiense inferior, y dentro del límite inferior del rango de medidas de Megacricetodon crusafonti.

En la localidad de Simancas 2, en la cuenca del Duero, se presenta un caso similar (Álvarez - Sierra \& García - Moreno, 1986; López et al., 1986). En este yacimiento aparecen juntas dos especies del género Megacricetodon. Por una parte, un conjunto de restos que adscriben a $\mathrm{Me}$ gacricetodon crusafonti - ibericus, y por otra, un conjunto de ejemplares de menor talla que determinan como la nueva especie Megacricetodon lopezae García Moreno, 1986. Según estos autores, Megacricetodon lopezae es de talla más reducida que Megacricetodon crusafonti - ibericus, y mayor que la que presentan las poblaciones de Megacricetodon minor.

Si comparamos el material de pequeña talla de Cazuma 1 con el de Simancas 2, podemos observar que, si bien las medidas son similares, la morfología es bastante diferente. En el $\mathrm{M}_{1}$ de Megacricetodon lopezae de Simancas 2 los mesolófidos están muy reducidos o ausentes, mientras que el anterocónido es puntiforme o ligeramente dividido. Igualmente, mientras el $\mathrm{M}_{1}$ de Cazuma 1 es alargado y estrecho, el $\mathrm{M}_{1}$ de Megacricetodon lopezae esta más redondeado.

Por todo lo expuesto, no podemos concluir una determinación más precisa de este resto. Por el momento, y a la espera de contar con nuevo material, preferimos determinar el resto como Megacricetodon sp.

Familia Sciuridae Gray, 1821

Tribu Xerini Osborn, 1910

Género Heteroxerus Stehlin \& Schaub, 1951

Especie tipo: Xerus grivensis Forsyth Major, 1893

\section{Heteroxerus aff. grivensis (Forsyth Major, 1893) Fig. 51}

Material y medidas: Un $\mathrm{P}^{4}$ de longitud $1,63 \mathrm{~mm}$ y una anchura de $1,77 \mathrm{~mm}$.

Descripción: $\mathbf{P}^{\mathbf{4}}$, anterolofo robusto y unido al paracono. Protolofo grueso. Protocono, paracono y metacono son las cúspides más altas. Metacono unido al metacónulo por un grueso metalofo. Metalofo incompleto, unido por la conexión longitudinal al posterolofo. Debido a esta unión y al cíngulo labial resultante del desarrollo del mesostilo, el valle central queda aislado.

\section{Discusión:}

La presencia de un $\mathrm{P}^{4}$ con metalofo incompleto, que une el metacono a un grueso metacónulo, junto al tamaño, nos permite encuadrar nuestro material en el género Heteroxerus Stehlin \& Schaub, 1951.

En algunas localidades del Aragoniense inferior y medio, Heteroxerus rubricati Crusafont, Villalta \& Truyols, 1955 y Heteroxerus grivensis (Forsyth Major, 1893) com- parten morfología general y talla (Cuenca, 1988). Esta autora establece como uno de los criterios diferenciadores para la distinción entre ambas especies la unión del metalofo y el posterolofo. Mientras en Heteroxerus rubrica$t i$ está unión a veces aparece y otras no, en Heteroxerus grivensis es prácticamente constante.

En el $\mathrm{P}^{4}$ de Cazuma 1 se observa la unión entre el metalofo y el posterolofo a través de una cresta longitudinal, por lo que adscribimos provisionalmente este resto de la familia Sciuridae a Heteroxerus aff. grivensis, aunque el material es escaso, y no se puede comprobar el rango de variabilidad de este carácter.

\section{CONCLUSIONES}

La serie de materiales cenozoicos que aflora en el cauce del Río Cazuma contiene niveles lacustre - palustres que presentan restos de micromamíferos fósiles. Uno de estos niveles, el siglado como Cazuma 1, ha cedido una colección en la que pueden distinguirse, hasta el momento, tres taxones de roedores, Megacricetodon crusafonti (Freudenthal, 1963), Megacricetodon sp., y Heteroxerus aff. grivensis (Forsyth Major, 1893). La morfología y biometría de la población de Megacricetodon crusafonti de Cazuma 1 son muy similares a las de otras poblaciones de la Península Ibérica donde aparece esta especie.

La conjunción de Megacricetodon crusafonti (Freudenthal, 1963), y la fauna acompañante determinada, nos permite datar este yacimiento como Aragoniense superior, biozona G2 de Daams et al. (1999), ampliando el rango de distribución de Megacricetodon crusafonti a las cuencas de Antepaís Bético, en la transición entre la Cordillera Ibérica y las Béticas en el sur de la provincia de Valencia.

\section{AGRADECIMIENTOS}

Queremos expresar nuestro agradecimiento a Pascual, Juana y María por su ayuda prestada durante el lavado del material. El trabajo de campo fue posible realizarlo gracias al proyecto de investigación, código 02-055 "Estudi de les faunes de micromamífers fòsils de les conques terciaries de la provincia de València" concedido por la "Institució Valenciana d'Estudis i Investigació”.

\section{BIBLIOGRAFÍA}

Álvarez-Sierra, M. A. \& García-Moreno, E. 1986. New Gliridae and Cricetidae from the middle and upper Miocene of the Duero Basin, Spain. Stvdia Geologica Salmanticensia, 22, 145-189.

Antunes, M. T. \& Mein, P. 1977. Contributions à la Paléontologie du Miocène moyen continental du Bassin du 
Tage. III. Mammifères-Póvoa de Santarém, Pero Filho et Choes (Secorio). Conclusions générales. Ciencias da Terra, 3, 143-165.

Crusafont, M., Villalta, J.F. de \& Truyols, J. 1955. El burdigaliense continental de la cuenca del Vallés - Penedés. I: Estratigrafía. II: Paleontología. Memorias y comunicaciones del Instituto Geológico de la Diputación Provincial de Barcelona, 12, 11-272.

Cuenca, G. 1988. Revisión de los Sciuridae del Aragoniense y del Rambliense en la fosa de Calatayud-Montalbán. In: Biostratigraphy and paleoecology of the Neogene micromammalian faunas from the Calatayud-Teruel Basin (Spain) (Ed. M. Freudenthal). Scripta Geologica, special issue 2, 1-116.

Daams, R. \& Freudenthal, M. 1981. Aragonian: the Stage concept versus Neogene Mammal Zones. Scripta Geologica, 62, 1-17.

Daams, R. \& Freudenthal, M. 1988. Cricetidae (Rodentia) from the type Aragonian; the genus Megacricetodon. In: Biostratigraphy and paleoecology of the Neogene micromammalian faunas from the Calatayud-Teruel Basin (Spain) (Ed. M. Freudenthal). Scripta Geologica, special issue 1, 39-132.

Daams, R., Van der Meulen, A. J., Alvarez-Sierra, M. A., Peláez-Campomanes, P., Calvo, J. P., Alonso-Zarza, M. A. \& Krijgsman, W. 1999. Stratigraphy and sedimentology of the Aragonian (Early to Middle Miocene) in its type area (North-Central Spain). Newsletter on Stratigraphy, 37, 103-139.

Fahlbusch, V. 1964. Die Cricetiden (Mammalia) der Oberen Süsswassermolasse Bayerns. Abhandlungen Bayerischen Akademie der Wissenschaften, 118, 1-136

Forsyth Major, C. J. 1893. On some Miocene squirrels, with remarks on the dentition and classification of the Sciurinae. Proceedings of Zoological Society of London, 179-215.

Freudenthal, M. 1963. Entwicklungsstufen der miozänen Cricetodontinae (Mammalia, Rodentia) Mittelspaniens und ihre stratigraphische Bedeutung. Beaufortia, 119 (10), 51-154.

Freudenthal, M. 1968. On the mammalian fauna of the Hipparion- beds in the Calatayud-Teruel Basin (Prov. Zaragoza, Spain). Part IV, the genus Megacricetodon (Rodentia). Koninkle Nederlanden Akademie van Wetenschappen, Series B, 71, 51-72.

Gutiérrez, G., Elizaga, E., Goy, J. L., Nieto, M. \& Robles, F. 1984. Mapa Geológico de la Província de Valencia. Diputación Provincial de Valencia, Valencia, 61 pp.
Lartet, E. 1851. Notice sur la colline de Sansan. Portes, Auch, 1-47.

López Martinez, N., García Moreno, E. \& Álvarez-Sierra, M. A. 1986. Paleontología y Bioestratigrafía (micromamíferos) del Mioceno medio y superior del sector central de la cuenca del Duero. Studia Geologica Salmanticensia, 22, 191-212.

Pérez, A., Azanza, B., Cuenca, G., Pardo, G. \& Villena, J. 1985. Nuevos datos estratigráficos y paleontológicos sobre el Terciario del borde meridional de la depresión del Ebro (Provincia Zaragoza). Estudios Geológicos, 41, 405-411.

Roca, E., Anadón, P., Utrilla, R. \& Vazquez, A. 1996. Rise, closure and reactivation of the Bicorp-Quesa evaporite diapir, eastern Prebetics, Spain. Journal of the Geological Society, 153, 311-321.

Ruiz Sánchez, F. J. 1999. Estudio paleontológico de los roedores fósiles del Mioceno inferior de la cuenca del Río Magro (Valencia). Memoria Tesis Doctoral. Universitat de València, 1-395 (inédito).

Ruiz-Sánchez, F. J., Santisteban, C. \& Lacomba, J. I. 1994. Edad de los depósitos inferiores de la Cuenca del Cabriel en la localidad Fuen Vich-1. In: Comunicaciones del $2^{\circ}$ Congreso del Grupo Español del Terciario (eds. A. Muñoz, A. González \& A. Pérez). Universidad de Zaragoza, 197-200.

Ruiz-Sánchez, F. J., Lacomba, J. I. \& Santisteban de, C. 1995. Caracterización de Megacricetodon collongensis (Mammalia, Rodentia) del Aragoniense de la localidad "Quesa 2" (Quesa, Valencia). Revista Española de Paleontología, 10, 151-160.

Santisteban, C., Ruiz-Sánchez, F. J. \& Lacomba, J. I. 1994. Estratigrafía, edad y evolución de los depósitos terciarios de la cuenca de antepaís de Quesa-Bicorp. In: Comunicaciones del $2^{\circ}$ Congreso del Grupo Español del Terciario (eds. A. Muñoz, A. González \& A. Pérez). Universidad de Zaragoza, 209-212.

Sesé, C., López, N. \& Herráez, E. 1985. Micromamíferos (Insectívoros, Roedores y Lagomorfos) de la provincia de Madrid. In: Geología y Paleontología del Terciario continental de la provincia de Madrid (Ed. M. T. Alberdi). Memorias del Museo Nacional de Ciencias Naturales - CSIC, Madrid, 29-40.

Stehlin, H. G. \& Schaub, S. 1951. Die Trigonodontie der simplicidentaten Nager. Schweizerische Palaeontologische Abhandlungen, 67, 1-385.

Manuscrito recibido: 14 de Enero, 2004 Manuscrito aceptado: 12 de Mayo, 2005 